\title{
INDUCCIÓN DE TOLERANCIA EN MODELO DE AUTOTRASPLANTE*
}

\author{
Drs. Alejandro E. Ramírez M. ${ }^{1}$, Mirentxu Iruretagoyena B. ${ }^{2}$, \\ Rodrigo Contreras D. ${ }^{3}$, Jaime Cartes U. ${ }^{4}$, Mónica Martínez M. ${ }^{5}$, \\ Carlos Martínez M. ${ }^{6}$, Valeria Alvarado S. ${ }^{6}$, Jorge Martínez C. ${ }^{7}$
}

1 Residente de Cirugía Plástica y Reconstructiva. División de Cirugía. Facultad de Medicina, Pontificia Universidad Católica de Chile.

2 Departamento de Inmunología Clínica y Reumatología. Facultad de Medicina, Pontificia Universidad Católica de Chile.

3 Clínica Lo Curro.

${ }^{4}$ Residente de Urología. División de Cirugía. Facultad de Medicina, Pontificia Universidad Católica de Chile.

5 Residente de Cirugía General. División de Cirugía. Facultad de Medicina, Pontificia Universidad Católica de Chile.

${ }^{6}$ Laboratorio de Cirugía Experimental, Centro de Investigaciones Médicas. División de Cirugía. Facultad de Medicina, Pontificia Universidad Católica de Chile.

7 Programa de Trasplantes, Departamento de Cirugía Digestiva. Facultad de Medicina, Pontificia Universidad Católica de Chile.

Santiago, Chile.

\section{Abstract}

\section{Induction of tolerance in allogeneic transplant model}

Introduction: Vascularized composite allotransplantation (VCA) involves the transplantation of complex anatomical structures including different kinds of tissue. The aim was to study the effect of a treatment with immature dendritic cells in a model of VCA. Materials and Methods: The rat hind limb allotransplantation model was used. Due to the high antigenic mistmatch Brown Norway rats were used as donors and Lewis rats as recipients. The bone marrow derived immature dendritic cells were cultured under GM-CSF stimuli and donor tissue. The rejection grade and the survival of the graft were assessed. Experimental groups: group I $(\mathrm{n}=3)$ : no treatment; Group II $(\mathrm{n}=6)$ : tacrolimus $10 \mathrm{mg} / \mathrm{kg}$ one day before the transplantation (day -1$)$; Group III $(\mathrm{n}=3)$ : tacrolimus $10 \mathrm{mg} / \mathrm{kg}$ on day -1 and $6 \mathrm{mg} / \mathrm{kg}$ from day 0 to 14 , plus intravenous saline

*Recibido el 28 de octubre de 2014 y aceptado para publicación el 23 de marzo de 2015.

Premio mejor trabajo de investigación 2011. Tercer Congreso Chileno de Trasplante. Sociedad Chilena de Trasplante.

Financiamiento: Proyecto de Residentes, División de Cirugía, Pontificia Universidad Católica de Chile.

Este estudio fue aprobado por el Comité de Ética y Bienestar Animal.

Los autores no refieren conflictos de interés.

Correspondencia: Dr. Jorge Martínez C. jamartin@med.puc.cl 
infusion on days 7 and 14; Group IV ( $\mathrm{n}=3$ ): tacrolimus $10 \mathrm{mg} / \mathrm{kg}$ on day -1 and $6 \mathrm{mg} / \mathrm{kg}$ from day 0 to 14 , plus intravenous immature dendritic cells on days 7 and 14. Results: All 15 allografts developed rejection. The mean allograft survival was 14 days in group I, 15 days in group II, 34 days in group III and 58 days in groups IV ( $p<0.05)$. Conclusions: In the rat hind limb allotransplantation model under tacrolimus monotherapy, the treatment with immature bone marrow derived dendritic cells pulsed with alloantigens increases the survival of the graft.

Key words: Allotransplantation, rejection, induction, dendritic cells, alloantigens.

\section{Resumen}

Introducción: El alotrasplante compuesto vascularizado (ACV) involucra el trasplante de estructuras anatómicas complejas que pueden contener distintos tipos de tejidos. El objetivo de este estudio fue evaluar el efecto del tratamiento con células dendríticas inmaduras derivadas de médula ósea del receptor y cargadas con aloantígenos como potencial inductor de tolerancia en un modelo de ACV. Animales y Métodos: Para realizar el modelo de alotrasplante de extremidad posterior de la rata, se utilizaron como donantes ratas Brown Norway y como receptoras ratas Lewis. Las células dendríticas se diferenciaron a partir de precursores de médula ósea que se cargaron con lisado de tejido del donante. Grupos experimentales: Grupo I $(n=3)$ : sin tratamiento; Grupo II $(\mathrm{n}=6)$ : tacrolimus $10 \mathrm{mg} / \mathrm{kg}$ vía oral el día previo al trasplante (día -1); Grupo III $(\mathrm{n}=3)$ : tacrolimus $10 \mathrm{mg} / \mathrm{kg}$ el día -1 y $6 \mathrm{mg} / \mathrm{kg}$ desde el día 0 al 14 post operatorio como mantención; Grupo IV $(\mathrm{n}=3)$ : mismo esquema de tacrolimus que grupo III, pero además infusión intravenosa de células dendríticas los días 7 y 14. Se evaluó la sobrevida de los implantes y el grado de rechazo. Resultados: Los 15 animales trasplantados presentaron rechazo. La sobrevida media del ACV fue de 14 días en el grupo I, 15 días en el grupo II, 34 días en el grupo III y 58 días en el grupo IV $(\mathrm{p}<0,05)$. Conclusión: En un modelo de ACV bajo tratamiento con tacrolimus, la infusión de células dendríticas inmaduras derivadas de médula ósea y pulsadas con aloantígeno aumentan la sobrevida del implante.

Palabras clave: Alotrasplante, rechazo, inducción, células dendríticas, aloantígenos.

\section{Introducción}

El alotrasplante compuesto vascularizado (ACV) consiste en el trasplante de tejidos complejos que pueden contener piel, músculos, nervios, vasos sanguíneos, tendones, huesos, y células inmunes, o una combinación de los anteriores ${ }^{1,2}$. El ACV tiene un potencial considerable de aplicación en el campo de la cirugía reconstructiva. Se estima que cada año en Estados Unidos, cerca de 7 millones de personas se podrían beneficiar de un $\mathrm{ACV}$, luego de la pérdida de tejidos por accidentes, cirugías oncológicas o malformaciones congénitas. Sin embargo, uno de sus principales problemas es la exposición de pacientes sin riesgo vital a los riesgos que la inmunosupresión crónica conlleva ${ }^{3-5}$. Como una aproximación para el estudio de esta nueva área de la medicina, se han descrito varios modelos experimentales, entre ellos el trasplante de extremidad posterior de la rata, considerado en la actualidad el estándar de los modelos animales de $\mathrm{ACV}^{6,7}$. El desarrollo de este y otros modelos ha permitido estudiar distintos aspectos relevantes del ACV, tales como la factibilidad técnica de estos trasplantes, las diferencias inmunológicas con los trasplantes de órganos sólidos y la recuperación motora y sensitiva de los implantes ${ }^{8-16}$. Diversos estudios han mostrado las diferencias en inmunogenicidad de los distintos tejidos que componen un ACV. De la misma forma, otros estudios han evidenciado cómo la cantidad y el tipo de tejido trasplantado influye en el grado de rechazo $^{17,18}$. Así mismo, estos modelos han permitido el estudio de medicamentos inmunosupresores, y últimamente el ensayo de tratamientos inductores de tolerancia, definida de forma operacional como la capacidad de prescindir del uso de inmunosupresores para mantener el implante libre de recha$\mathrm{zo}^{19}$. Estudios previos han sugerido la utilidad del tratamiento con células dendríticas como inductor de tolerancia en modelos de trasplante de órganos sólidos y en modelos de $\mathrm{ACV}^{20-31}$. Sin embargo, ninguno de estos trabajos ha estudiado si las células dendríticas derivadas de médula ósea son efectivas como inductor de tolerancia, en un modelo que tenga como base de tratamiento inmunosupresor (Tis) al tacrolimus, que es la base del tratamiento actual en ACV clínico.

El objetivo de este estudio fue montar un modelo descrito de trasplante de tejidos compuestos, el alotrasplante de extremidad posterior de la rata, para evaluar el tratamiento con células dendríticas inmaduras cargadas con aloantígenos del donante. 


\section{Materiales y Métodos}

Este estudio fue aprobado por el Comité de Ética y Bienestar Animal (CEBA), del Centro de Investigaciones Médicas de la Facultad de Medicina de la Pontificia Universidad Católica de Chile.

\section{Animales y Métodos}

\section{Animales}

Dado su alto mismatch antigénico se utilizaron como donantes ratas macho Brown Norway (BN), y como receptores ratas Lewis, con edades entre 9 y 16 semanas y peso entre 180 y $330 \mathrm{~g}$.

\section{Cuidados pre y postoperatorio}

Los animales fueron mantenidos en jaulas individuales, con ciclo luz-oscuridad de $12 \mathrm{~h}$ y alimentación ad libitum. Todos los animales en el postoperatorio recibieron como analgésico una dosis diaria de ketoprofeno $5 \mathrm{mg} / \mathrm{kg} /$ día sc, por 3 días. Además recibieron enrofloxacino como antibiótico profiláctico $5 \mathrm{mg} / \mathrm{kg} /$ día hasta completar 3 dosis.

\section{Anestesia}

Como anestésico se utilizó una mezcla de ketamina $(70 \mathrm{mg} / \mathrm{kg})$ y xilazina $(3 \mathrm{mg} / \mathrm{kg})$ como inductor. Para la mantención se utilizaron bolos de ketamina $(23 \mathrm{mg} / \mathrm{kg})$, ambos por vía intraperitoneal (ip). Durante la cirugía se utilizó un calentador eléctrico para evitar la hipotermia de los animales. Recibieron además $2 \mathrm{ml}$ de suero $\mathrm{NaCl} 0,9 \%$ ip, para disminuir el riesgo de hipovolemia.

\section{Análisis estadístico}

Las variables cuantitativas continuas se expresaron como media \pm desviación estándar o como mediana y rango. Las variables cuantitativas discretas y cualitativas se expresaron como porcentajes. Para el análisis de sobrevida se realizaron curvas de KaplanMeier y Log Rank test.

\section{Trasplante de extremidad posterior de la rata}

Una vez realizado un programa de entrenamiento en microcirugía ${ }^{32}$, se dio inicio a la serie de trasplantes. Todos los trasplantes fueron realizados por el mismo cirujano A.E.R ${ }^{1}$. Por razones éticas y para disminuir el número de animales necesarios para los trasplantes, cada rata $\mathrm{BN}$ fue utilizada como donante de ambas extremidades posteriores y cada rata Lewis como receptora de una extremidad. Los trasplantes se realizaron según modificación de la técnica descrita por $\mathrm{Doi}^{7}$.

\section{Cirugía de procura}

Bajo anestesia general, en las ratas $\mathrm{BN}$ se procuraron las extremidades posteriores. Resumidamente, se realizó sección de vasos femorales, sección de grupos musculares del muslo, sección del nervio ciático y osteotomía a nivel medio del fémur, protegiendo los vasos femorales de cualquier traumatismo (Figura 1).

Las extremidades extraídas fueron mantenidas en bolsas estériles, sin contacto directo con hielo, y almacenadas a $4^{\circ} \mathrm{C}$ hasta el inicio del implante.

\section{Cirugía de preparación del receptor}

Para la preparación de las ratas Lewis, se amputó la extremidad a reemplazar con una técnica similar a la descrita en la procura, con la diferencia que el control proximal de los vasos femorales se realizó con microclamps, y la sección de los vasos se realizó lo más distal posible, inmediatamente proximal al origen de los vasos epigástricos superficiales.

\section{Cirugía de implante}

El implante en las ratas Lewis se realizó según la siguiente secuencia: Osteosíntesis del fémur con una aguja $21 \mathrm{~g}$ a modo de clavo endomedular más cerclaje simple con alambre de acero quirúrgico de $0,4 \mathrm{~mm}$ (Figura 2). Neurorrafía del nervio ciático con 4 puntos epineurales de Nylon 10-0. Afrontamiento de los distintos grupos musculares con sutura interrumpida de Prolene 5-0. Anastomosis venosa femoral terminoterminal con sutura interrumpida de Nylon 10-0 con técnica de triangulación (Figura 3). Anastomosis de arteria femoral termino-terminal con sutura interrumpida de Nylon 10-0 con técnica de pared anterior y pared posterior. Ambas anastomosis se realizaron bajo irrigación directa de heparina no fraccionada $100 \mathrm{UI} /$ $\mathrm{ml}$. Afrontamiento de la piel con nylon 3-0.

\section{Tratamiento inmunosupresor y evaluación del rechazo}

Como Tis se utilizó tacrolimus en forma de monoterapia. Nuestro objetivo fue mantener a los

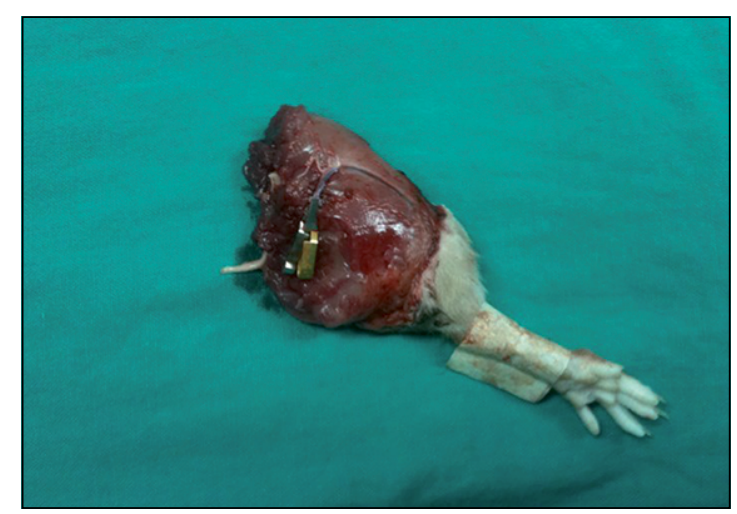

Figura 1. Extremidad posterior procurada de rata Brown Norway. 


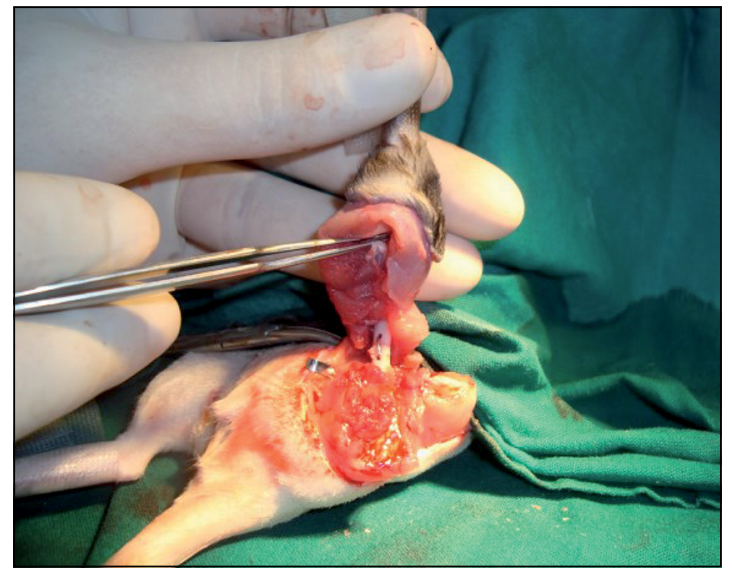

Figura 2. Implante de extremidad posterior izquierda. Se observa la fijación ósea del fémur y los músculos del muslo.

animales libres de rechazo hasta poder utilizar el tratamiento con células dendríticas.

La intensidad del rechazo se evaluó macroscópicamente por dos observadores independientes no ciegos con una escala visual: Grado $(G) 0$ : $\sin$ rechazo; GI: sospecha de rechazo; GII: rechazo leve; GIII: rechazo moderado; GIV: rechazo grave ${ }^{33}$. En los casos de rechazo GI-GIII, se confirmó el infiltrado linfocitario dermoepidérmico con biopsias de piel evaluadas con tinción de H\&E. Para el cálculo de la sobrevida del injerto, se consideró como pérdida del injerto cuando clínicamente se observara más de un $90 \%$ de necrosis del implante. Terminado el período de observación la eutanasia de los animales se realizó según protocolo del CEBA.

\section{Tratamiento con células dendríticas}

Una vez lograda la mantención de los animales libres de rechazo por al menos 1 semana se comenzó con la infusión intravenosa (iv) de células dendríticas. Los cultivos de células dendríticas se realizaron según modificación de la técnica descrita por Feili-Hariri et $\mathrm{al}^{34}$. De forma resumida, para la preparación de la infusión de células dendríticas se realizaron cultivos de médula ósea obtenida del fémur y la tibia de ratas LW. El cultivo se estimuló con factor estimulador de colonia de granulocitos y macrófagos (GM-CSF). Se evaluó por citometría de flujo con marcadores específicos para células dendríticas, lográndose una diferenciación mayor al 80\% de células dendríticas en el cultivo. A los 6 días de cultivo se cargaron las células dendríticas con tejido del donante $(\mathrm{BN})$. Como tejido del donante se utilizó un lisado de músculo del muslo del donante. Se agregó $1 \mathrm{ml}$ de lisado a la placa de cultivo de células

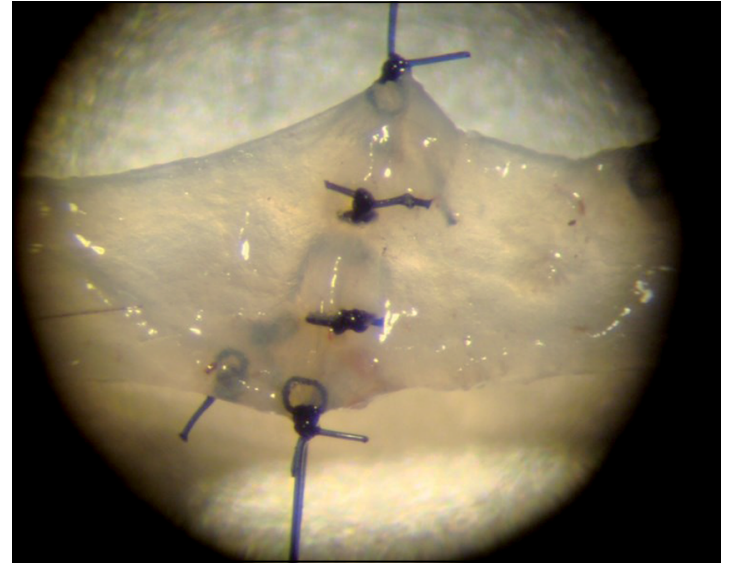

Figura 3. Anastomosis microvascular termino-terminal de vena femoral. Técnica de triangulación con nylon 10.0 .

dendríticas durante $24 \mathrm{~h}$. Cada infusión contenía $7 \mathrm{x}$ $10^{6}$ células dendríticas cargadas con aloantígeno, en un volumen de $0,5 \mathrm{ml}$. Se administró una infusión iv el día 7 y una segunda infusión el día 14 postoperatorio.

\section{Grupos experimentales de ratas con alotrasplante de extremidad posterior}

Grupo I $(\mathrm{n}=3)$ : sin tratamiento.

Grupo II $(\mathrm{n}=6)$ : tacrolimus $10 \mathrm{mg} / \mathrm{kg}$ vía oral por gavage el día previo al trasplante (día -1).

Grupo III $(\mathrm{n}=3)$ : tacrolimus $10 \mathrm{mg} / \mathrm{kg}$ vo el día -1 y $6 \mathrm{mg} / \mathrm{kg}$ vo desde el día 0 al 14 post operatorio (po) como mantención. Además infusión iv de suero fisiológico $0,9 \%(0,5 \mathrm{cc})$ los días 7 y 14 po.

Grupo IV $(\mathrm{n}=3)$ : tacrolimus $10 \mathrm{mg} / \mathrm{kg}$ vo el día -1 y $6 \mathrm{mg} / \mathrm{kg}$ vo desde el día 0 al 14 po. Además infusión iv de células dendríticas $(0,5 \mathrm{cc})$ los días 7 y 14 po.

\section{Resultados}

Los 15 animales trasplantados sobrevivieron en buenas condiciones durante todo el período del estudio.

\section{Tratamiento inmunosupresor}

Todos los animales sin Tis (grupo I) desarrollaron rechazo antes del día 6 (Figura 4). En el grupo II no se logró un aumento significativo en la sobrevida libre de rechazo con una dosis única de tacrolimus $(\mathrm{p}>0,05)$. El grupo III y IV con dosis diaria de tacrolimus se logró mantener todos los animales libres de signos de rechazo hasta el día $7(\mathrm{p}<0,05)$ (Figura 5). 


\section{Tratamiento con células dendríticas}

Luego de la suspensión del Tis con tacrolimus el día 14 po, todos los animales del grupo III y IV desarrollaron rechazo. La Figura 6 muestra la comparación de sobrevidas de los ACV entre el grupo III y IV. La sobrevida media en el grupo IV fue de 58 días versus 34 días en el grupo III $(\mathrm{p}<0,05)$.

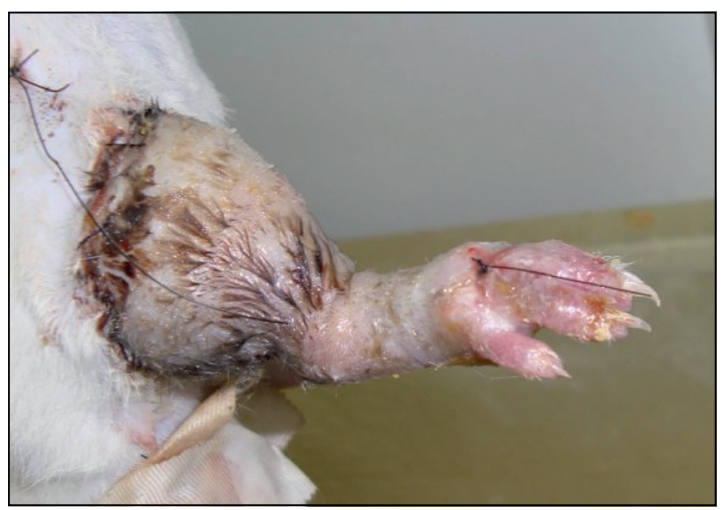

Figura 4. Alotrasplante de extremidad posterior de la rata, grupo 2 (dosis única de tacrolimus), día 9 postoperatorio. Rechazo moderado (grado III). Se observa el engrosamiento difuso de la piel y la pérdida parcial de epidermis (sobre los dedos), además de cambios en el color de la extremidad (áreas blanquecinas y áreas con máculas).

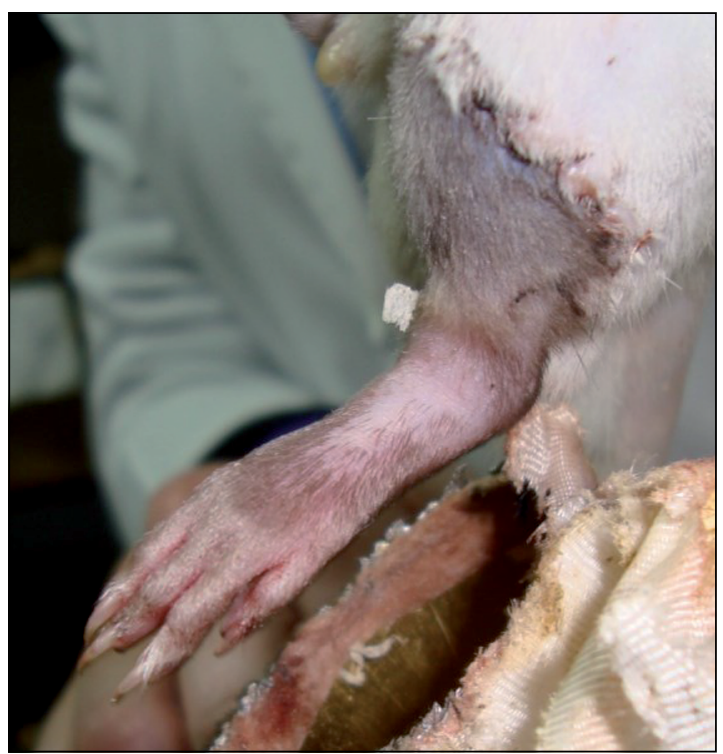

Figura 5. Alotrasplante de extremidad posterior de la rata grupo 3 (dosis diaria de tacrolimus), día 7 postoperatorio. Sin rechazo (grado 0). Se observa la epidermis intacta. No hay engrosamiento de la piel ni cambios de color respecto a una extremidad normal. Se observa además el crecimiento de los pelos de la extremidad.

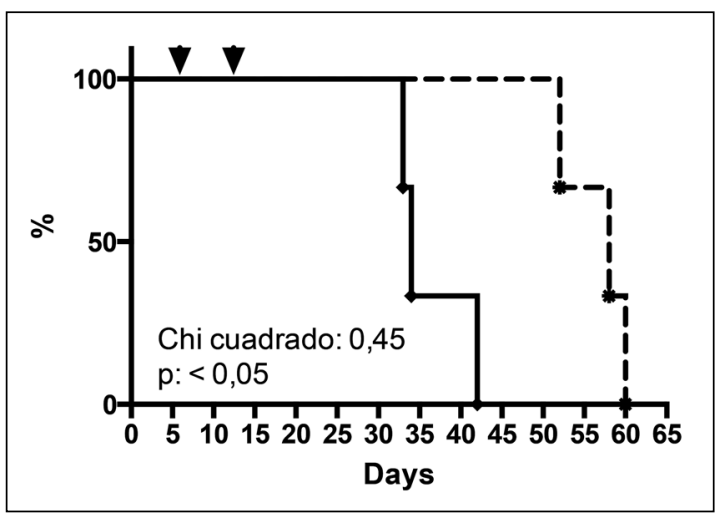

Figura 6. Sobrevida del alotrasplante de extremidad posterior de la rata (\%). La línea negra continua muestra el grupo III (tratamiento con tacrolimus hasta día 14 po, más infusión iv de suero fisiológico los días 7 y 14 po). La línea negra discontinua muestra el grupo IV (tratamiento con tacrolimus hasta día 14 po, más infusión iv de células dendríticas los días 7 y 14 po. La diferencia en la sobrevida del implante fue estadísticamente significativa (p: <0,05). Las cabezas de flechas muestran los días de las infusiones (día 7 y 14 postoperatorio).

\section{Discusión}

Los datos históricos sobre trasplante de extremidad se remontan al siglo 3 d.C. Según la leyenda, San Cosme y San Damián trasplantaron la extremidad de un etíope fallecido a uno de sus pacientes que sufría gangrena en una de sus piernas. Varios años pasaron hasta que el desarrollo de la medicina y la cirugía permitieron en 1964 realizar el primer trasplante de mano en Ecuador ${ }^{35}$. Sin embargo, por esos días no se tenía los conocimientos ni el armamentario terapéutico para contrarrestar el rechazo subsecuente. El implante tuvo que ser amputado semanas después de la cirugía. No fue sino hasta el descubrimiento de la ciclosporina y su uso en distintos trasplantes de órganos sólidos, que comenzó nuevamente a considerarse la posibilidad de realizar alotrasplantes reconstructivos. Los ACV más emblemáticos a la fecha son el primer trasplante de mano exitoso realizado en Francia el año $1998^{36}$ y el primer trasplante de cara realizado en el mismo país el $2005^{37}$. Sin embargo, a pesar de los avances alcanzados, aún no se solucionan problemas como la necesidad de inmunosupresores, y la sub-óptima recuperación funcional alcanzada en algunos casos. Es en estas y en otras áreas donde los modelos de ACV cumplen su mayor función, al permitirnos estudiar distintos aspectos relevantes del $\mathrm{ACV}^{14}$. Creemos que para poder realizar estos modelos microquirúrgicos complejos, muchas veces con tiem- 
pos operatorios prolongados, es fundamental poner cuidado en la anestesia y los cuidados generales de los animales. Un entrenamiento microquirúrgico básico ${ }^{32}$, nos parece recomendable para optimizar los recursos y el número de animales necesarios para realizar la investigación.

Si bien existen estudios con esquemas mixtos de tratamiento inmunosupresor, usar una monoterapia de base parece ser una mejor opción para comparar el efecto de tratamientos inductores de tolerancia. Esto con el objetivo de disminuir los costos y simplificar el tratamiento.

Sacks et al. ${ }^{31}$, en un modelo de trasplante de extremidad posterior de rata Wistar-furt a Lewis, demostró que el tratamiento con células dendríticas aumenta la sobrevida de los implantes al comparar dos grupos que tenían como base de tratamiento ciclosporina y suero anti-linfocítico. Nguyen et al. ${ }^{29}$, demostraron que las células dendríticas derivadas de médula ósea cargadas con alopéptidos aumentan la sobrevida del implante en un modelo de trasplante de extremidad de la rata bajo terapia con suero anti-linfocítico. En el estudio de Eun et al. ${ }^{34}$, no fue posible inducir tolerancia clínica en los animales que recibieron tratamiento con células dendríticas inmaduras derivadas de células sanguíneas mononucleares periféricas. En el presente trabajo, se logró un aumento significativo en la sobrevida del implante al suspender el Tis, sin embargo, no logramos demostrar tolerancia. Estos hallazgos son clínicamente relevantes ya que el tacrolimus es actualmente la base del Tis en pacientes con ACV. Este trabajo y el de Eun et al. muestran que el efecto de las células dendríticas y su capacidad de modular la respuesta inmune están presentes en un ACV que tiene como base de tratamiento tacrolimus. Si bien estos resultados parecen prometedores aún quedan interrogantes por dilucidar, tales como el número de infusiones necesarias, el número de células de cada infusión, el efecto de los distintos Tis sobre la maduración y la actividad presentadora de antígeno e inductora de tolerancia de las células dendríticas inmaduras in vivo. Eventualmente las respuestas a estas preguntas llevarán al tratamiento con células dendríticas a tener una potencial aplicabilidad clínica, manteniendo a los pacientes con ACV libre de los riesgos que el tratamiento crónico con inmunosupresores conlleva.

\section{Referencias}

1. Kann BR, Furnas DW, Hewitt CW. Past, present and future research in the field of composite tissue allotransplantation. Microsurgery 2000;20:393-9.

2. Swearingen B, Ravindra K, Xu H, Wu S, Breidenbach WC, Ildstad ST. Science of composite tissue allotransplantation. Transplantation 2008;86:627-35.
3. Jablecki J, Kaczmarzyk L, Patrzalek D, Chelmonski A, Domanasiewicz A. Mild rejection episode evoked by modification of immunosuppressive protocol observed in a patient with forearm transplantation: case report. Transplant Proc. 2009;41:557-9.

4. Schneeberger S, Gorantla VS, Hautz T, Pulikkottil B, Margreiter R, Lee WP. Immunosuppression and rejection in human hand transplantation. Transplant Proc. 2009; 41:472-5.

5. Schneeberger S, Landin L, Kaufmann C, Gorantla VS, Brandacher G, Cavadas P, et al. Alemtuzumab: key for minimization of maintenance immunosuppression in reconstructive transplantation? Transplant Proc. 2009;41:499-502.

6. Shapiro RI, Cerra FB. A model for reimplantation and transplantation of a complex organ: the rat hind limb. J Surg Res. 1978;24:501-6.

7. Doi K. Homotransplantation of limbs in rats. A preliminary report on an experimental study with nonspecific immunosuppressive drugs. Plast Reconstr Surg. 1979;64:613-21.

8. Lao WW, Wang YL, Ramírez AE, Cheng HY, Wei FC. A new rat model for orthotopic abdominal wall allotransplantation. Plastic and reconstructive surgery Global open 2014;2:e136.

9. Santiago SF, de Faria W, Khan TF, Gandia CE, Misiakos EP, Ferrer L, et al. Heterotopic sternum transplant in rats: A new model of a vascularized bone marrow transplantation. Microsurgery 1999;19:330-4.

10. Liao TC, Yeh LS, Chu RM, Hou SM. An osteomyocutaneous transplantation model on the rat. Microsurgery 2001;21:329-32.

11. Ulusal BG, Ulusal AE, Ozmen S, Zins JE, Siemionow MZ. A new composite facial and scalp transplantation model in rats. Plast Reconstr Surg. 2003;112:1302-11.

12. Ulusal AE, Ulusal BG, Hung LM, Wei FC. Heterotopic hindlimb allotransplantation in rats: an alternative model for immunological research in composite-tissue allotransplantation. Microsurgery 2005;25:410-4.

13. Yazici I, Unal S, Siemionow M. Composite hemiface/ calvaria transplantation model in rats. Plast Reconstr Surg. 2006;118:1321-7.

14. Siemionow M, Kulahci Y. Experimental models of composite tissue allograft transplants. Semin Plast Surg. 2007;21:205-12.

15. Nie C, Yang D, Li N, Liu G, Guo T. Establishing a new orthotopic composite hemiface/calvaria transplantation model in rabbits. Plast Reconstr Surg. 2008;122:410-8.

16. Horner BM, Randolph MA, Duran-Struuck R, Hirsh EL, Ferguson KK, Teague AG, et al. Induction of tolerance to an allogeneic skin flap transplant in a preclinical large animal model. Transplant Proc. 2009;41:539-41.

17. Ramírez AE, Cheng HY, Lao WW, Wang YL, Wen CJ, Wallace CG, et al. A novel rat full-thickness hemiabdominal wall/hindlimb osteomyocutaneous combined flap: influence of allograft mass and vascularized bone 
marrow content on vascularized composite allograft survival. Transpl Int. 2014;27:977-86.

18. Ulusal BG, Ulusal AE, Wei FC, Lin CY. Allograft mass as a possible contributing factor to the skin transplant outcome. J Surg Res. 2010;161:321-7.

19. Starzl TE, Murase N, Abu-Elmagd K, Gray EA, Shapiro R, Eghtesad B, et al. Tolerogenic immunosuppression for organ transplantation. Lancet 2003;361:1502-10.

20. Chiang YJ, Lu L, Fung JJ, Qian S. Liver-derived dendritic cells induce donor-specific hyporesponsiveness: use of sponge implant as a cell transplant model. Cell Transplant. 2001;10:343-50.

21. Auffermann-Gretzinger S, Lossos IS, Vayntrub TA, Leong W, Grumet FC, Blume KG, et al. Rapid establishment of dendritic cell chimerism in allogeneic hematopoietic cell transplant recipients. Blood 2002;99:1442-8.

22. Coates PT, Barratt-Boyes SM, Donnenberg AD, Morelli AE, Murphey-Corb M, Thomson AW. Strategies for preclinical evaluation of dendritic cell subsets for promotion of transplant tolerance in the nonhuman primate. Hum Immunol. 2002;63:955-65.

23. Comoli P, Basso S, Azzi A, Moretta A, De Santis R, Del Galdo F, et al. Dendritic cells pulsed with polyomavirus $\mathrm{BK}$ antigen induce ex vivo polyoma BK virus-specific cytotoxic T-cell lines in seropositive healthy individuals and renal transplant recipients. J Am Soc Nephrol. 2003; 14:3197-204.

24. Porta MD, Rigolin GM, Alessandrino EP, Maiocchi M, Malcovati L, Vanelli L, et al. Dendritic cell recovery after allogeneic stem-cell transplantation in acute leukemia: correlations with clinical and transplant characteristics. Eur J Haematol. 2004;72:18-25.

25. Sumpter TL, Lunz Iii JG, Castellaneta A, Matta B, Tokita D, Turnquist HR, et al. Dendritic cell immunobiology in relation to liver transplant outcome. Front Biosci (Elite Ed). 2009;1:99-114.

26. Thomson AW, Sacks JM, Kuo YR, Ikeguchi R, Horibe EK, Unadkat J, et al. Dendritic cell therapy in composite tissue allotransplantation. Transplant Proc. 2009;41:5378.

27. Thomson AW, Turnquist HR, Zahorchak AF, Raimondi G. Tolerogenic dendritic cell-regulatory T-cell interac- tion and the promotion of transplant tolerance. Transplantation 2009;87:S86-90.

28. Ueda A, Ito M, Ishiguro N, Miura Y, Asai J, Miura T. Thy-1 antigen dendritic cells and rejection of composite tissue limb allografts in rats. Transplantation 1993;56:124-7.

29. Nguyen VT, Taieb A, Sacks JM, Unadkat JV, Clavijo JA, Kim H, et al. Allopeptide-pulsed dendritic cells and composite tissue allograft survival. Microsurgery 2007;27:105-11.

30. Horibe EK, Sacks J, Unadkat J, Raimondi G, Wang Z, Ikeguchi R, et al. Rapamycin-conditioned, alloantigenpulsed dendritic cells promote indefinite survival of vascularized skin allografts in association with T regulatory cell expansion. Transpl Immunol. 2008;18:307-18.

31. Sacks JM, Kuo YR, Taieb A, Breitinger J, Nguyen VT, Thomson AW, et al. Prolongation of composite tissue allograft survival by immature recipient dendritic cells pulsed with donor antigen and transient low-dose immunosuppression. Plast Reconstr Surg. 2008;121:37-49.

32. Ramírez AE, Contreras R, Cartes J, Martínez M, Martínez C, Alvarado V, et al. Basic Microsurgical Training for a Vascularized Composite Allotransplantation Animal Model. Rev Chil Cir. 2013;65:389-95.

33. Zamfirescu DG, Owen E, Lascar I, Molitor M, Zegrea I, Popescu M, et al. Sentinel skin allograft-a reliable marker for monitoring of composite tissue transplant rejection. Transplant Proc. 2009;41:503-8.

34. Feili-Hariri M, Kao H, Mietzner TA, Morel PA. Functional consequences of the binding of MHC class II-derived peptides to MHC class II. Int Immunol. 1996;8:1857-65.

35. Piza-Katzer H, Wechselberger G, Estermann D, Gabl M, Arora R, Hussl H. [Ten years of hand transplantation experiment or routine?]. Handchir Mikrochir Plast Chir. 2009;41:210-6.

36. Dubernard JM, Owen E, Herzberg G, Lanzetta M, Martin X, Kapila H, et al. Human hand allograft: report on first 6 months. Lancet 1999;353:1315-20.

37. Devauchelle B, Badet L, Lengele B, Morelon E, Testelin S, Michallet M, et al. First human face allograft: early report. Lancet 2006;368:203-9. 